\title{
El proyecto ALFINTRA: desarrollo y evaluación de las competencias informacionales en el Grado de Traducción e Interpretación
}

\author{
The ALFINTRA project: developing and evaluating information competences
} in the undergraduate studies in translation and interpreting

\author{
María PINTo \\ Universidad de Granada, e-mail: mpinto@ugr.es
}

\begin{abstract}
Resumen
Infolitrans es un portal concebido para analizar, explorar y mejorar las competencias en información de los estudiantes de traducción y de todos aquellos profesionales relacionados con esta área. Desde una perspectiva eminentemente practica, se ofrece una herramienta que permitirá no solo medir las distintas capacidades y aptitudes de carácter informativo del alumno, sino hacer un diagnostico de las mismas y resolver los problemas que se detecten en el desarrollo del programa. De esta manera se ofrece un instrumento de análisis, de evaluación, pero también de resolución de problemas relacionados con la información
\end{abstract}

Palabras clave: Competencias informacionales. Competencias digitales. Traducción e interpretación. Evaluación. Autoaprendizaje. Aprendizaje supervisado. E-learning.

\section{Introducción}

La Educación Superior debe posibilitar una capacitación que favorezca la interpretación de la información y la generación del propio conocimiento. Esta educación, dentro del marco del Espacio Europeo de Educación Superior (EEES), está basada en el aprendizaje del estudiantado y en el diseño de herramientas metodológicas que favorezcan la adquisición de habilidades y estrategias en relación a la gestión, el análisis, la evaluación y la recuperación de la información. En este sentido, en el informe coordinado por Delors para la Unesco (1996) se afirma que en el siglo XXI, la educación se ve obligada a proporcionar las cartas náuticas de un mundo complejo y en perpetua agitación y, al mismo tiempo, la brújula para moverse por ellas.

El actual potencial informativo de Internet, si bien es una ventaja en sí, para el estudiante se convierte en un espacio de incertidumbre a la hora de saber seleccionar adecuadamente la información de calidad para su proceso de

\begin{abstract}
Infolitrans is a portal designed to analyze, explore and improve the information competences of the students of translation and related areas. From a very practical pespective, the portal offers a tool that allows to measure the acquisition of information competences and aptitudes by the students, and also to diagnose them and to solve the problems that have been detected in the learning process. In this way, a learning tool is offered that is suitable for analysis and assessment, and also for problem solving in the field of information abilities acquisition.
\end{abstract}

Keywords: Information competences. Digital competences. Translation and intepretation. Assessment. Self-learning. Supervised learning. E-learning.

aprendizaje. A esto se une la transitoriedad, la falta de organización y la ausencia de valoración de muchos de los contenidos existentes en la red, dificultando aún más la tarea de navegar en este océano informativo salvaje y caótico. En el actual contexto globalizado de información y en el marco del paradigma educativo de la alfabetización informacional (ALFIN) es necesario que haya un mejor tratamiento, organización y sistematización de los recursos, en un entorno mediático cambiante, donde la información es creciente, compleja y en la mayoría de los casos dispersa. El estudiante se encuentra con dificultades para acceder a la información debido al gran número de recursos disponibles y a la poca estructuración de éstos. Necesitan nuevas estrategias que les permitan ser autosuficientes en los procesos de aprendizaje y adquirir competencias informacionales para detectar y definir una necesidad de información; expresarla y delimitarla; conocer los métodos y técnicas adecuadas para la localización de información; evaluar y filtrar la información, para asegurarse de 
su autenticidad, validez y fiabilidad y agregar dicha información a la base de conocimientos, así como utilizarla para afrontar objetivos de forma ética y legal (ACRL, 2000).

\section{La importancia de ALFIN como puente para el aprendizaje basado en competencias}

Los nuevos modelos educativos basados en competencias y habilidades están reemplazando a los antiguos modelos basados en la enseñanza del profesorado y el estudio pasivo por parte del alumnado. En el aprendizaje por competencias, el estudiante se convierte en protagonista activo de un aprendizaje virtual, interactivo, compartido y distribuido. La adquisición de destrezas en habilidades relacionadas con el uso y gestión de la información y las tecnologías le permitirá afrontar las competencias específicas de su área de conocimiento. Este cambio del proceso educativo, que supone el paso de una educación centrada en la enseñanza a una educación centrada en el aprendizaje, implica un nuevo enfoque en el papel de los educadores y de las actividades educativas, y da un mayor énfasis a los resultados de aprendizaje. El aprendizaje basado en competencias refleja la capacidad del estudiante para poner en práctica de manera integrada habilidades, conocimientos y actitudes para enfrentarse y poder resolver problemas y situaciones (OECD, 2005). Además, permiten evaluar su grado de preparación, suficiencia y responsabilidad. Sirvan de ejemplo los proyectos DESECO, TUNING, AlfinEEES.

Las competencias informacionales están relacionadas con la información (búsqueda, selección, valoración, análisis, procesamiento, comunicación...) y son imprescindibles en la Sociedad de la Información y del Conocimiento, caracterizada por un fácil acceso y uso masivo de información. Las posibilidades con las que cuenta un estudiante para acceder a la información son ingentes, desde Internet a los distintos sistemas de información, pero para aprovechar esta situación debe estar en posesión de una serie de competencias y habilidades informacionales que le permitan hacer un uso adecuado de los recursos de aprendizaje.

Las competencias tecnológicas hacen referencia al uso de las nuevas tecnologías en los procesos de aprendizaje. Debido a su desarrollo en los últimos años y a las posibilidades que ofrecen en el ámbito de la educación (e-learning, plataformas digitales...), se hace necesario capacitar a los estudiantes para que saquen el máximo partido de estas tecnologías, incor- porándolas a sus procesos de aprendizaje. El uso del correo electrónico, la difusión de información en Internet, el uso de programas informáticos específicos (de dibujo, de estadística, de presentación de información, simuladores....), la capacidad para instalar aplicaciones informáticas, etc. son algunas de las habilidades tecnológicas con las que debe contar un alumno para poder defenderse en este entorno tecnológico. Muchas de estas competencias tienen relación directa con las competencias informacionales, ya que Internet se ha convertido en el mayor canal de información y gran parte de la información que se produce se difunde en soporte electrónico.

La alfabetización informacional (ALFIN) se refiere a la capacidad del estudiante para utilizar de manera adecuada la información contenida en documentos y recursos electrónicos (buscar, valorar, gestionar, comunicar... información), en el que se integran las tecnologías de la información y la comunicación y los planteamientos elearning cimentados en la alfabetización digital. Si tenemos en cuenta que la alfabetización en información (ALFIN) tiene como objetivo crear personas capaces de resolver necesidades de información, encontrando, evaluando y usando la información para resolver problemas o tomar decisiones, entonces podemos afirmar que la ALFIN se configura como canal esencial y soporte imprescindible para el cambio pedagógico centrado en el estudiante por el que se aboga en el EEES, siendo necesario poder evaluar y medir los resultados de los estudiantes universitarios en cuanto al dominio de competencias informacionales.

\section{EI modelo INFOLITRANS para la alfabetización informacional}

Consideramos la traducción, en gran medida, como una operación de análisis de contenido, en el contexto de la comunicación humana, de los nuevos medios de comunicación y del papel que éstos desempeñan en la transmisión de información. Desde este punto de vista estructural, el contenido tiene en cuenta los canales y las limitaciones de los flujos de información, los procesos de comunicación, y sus funciones sociales. En general los procesos de traducción se desarrollan en dos etapas fundamentales, una primera ascendente, o de interpretación de datos; y una segunda descendente, de enunciación de esos datos interpretados en un nuevo contexto lingüístico cultural (Pinto, 2001).

En todo proceso de transferencia, quien traduce, junto con el resto de competencias que configuran la denominada competencia traductora, 
tendrá que desarrollar una competencia documental o competencia informacional, es decir, una capacidad para documentarse, basada en conocimientos, habilidades y valores. Así, autores como Vienne (2000) otorgan enorme importancia a la competencia documental en el marco de la competencia traductora, postulando que las habilidades más relevantes de quien traduce consisten en primer lugar en saber analizar la situación de traducción y en segundo lugar en saber elaborar una estrategia de documentación que se adecue a esa situación de traducción.

Sin duda, la competencia documental resulta esencial en todo el proceso formativo del traductor, desde el inicio, como parte de uno de los básicos objetivos generales de aprendizaje que define Hurtado (1996) para la enseñanza de la traducción: la asimilación de los principios fundamentales del estilo de trabajo del traductor profesional.

De hecho, la opinión de que la competencia documental planea sobre varias de las subcompetencias delineadas para configurar la macrocompetencia traductora, se ve ratificada por el Informe auspiciado por ANECA (2004) para la propuesta del nuevo Grado en Traducción e Interpretación. Destaca la importancia en lo relativo a la Documentación en la formación de traductores, enfatizando la necesidad de poseer formación para la gestión conceptual y tecnológica de la información y la documentación. La capacidad para la gestión de la información destaca como una de las principales competencias transversales genéricas. También en el conjunto de competencias específicas, que el informe clasifica en disciplinares (saber), profesionales (saber hacer) y académicas, se enfatiza que entre las competencias específicas que requiere el traductor e intérprete está la destreza para la búsqueda de información y la Documentación (Sales, 2006).

En suma, la relevancia de la necesidad de la formación documental en el terreno de la formación de traductores es indudable, desde el enfoque estratégico que hemos postulado y que también es visible en las propuestas de competencia traductora que destacan en España. En nuestra opinión, la alfabetización informacional puede ser en gran medida la matriz y sustento de la base de esa competencia estratégica que ha de estar presente, de forma esencial, en toda (macro)competencia traductora.

En esta línea, hemos elaborado una propuesta conceptual que da cuerpo a nuestro enfoque didáctico (Pinto, Sales, 2008): el modelo ALFINTRA, centrado en la alfabetización informacional para traductores, conocido en inglés por su acrónimo Infolitrans. Se articula en torno a tres ejes interrelacionados: facetas, competencias y habilidades. Las cuatro facetas en que se sustenta son: el conocimiento, las tecnologías, los recursos y los procesos, que desde una propuesta de ALFIN aplicada se correlacionan con competencias y habilidades cognitivas, informativo-digitales, comunicativas y estratégicas, respectivamente (Figura 1).

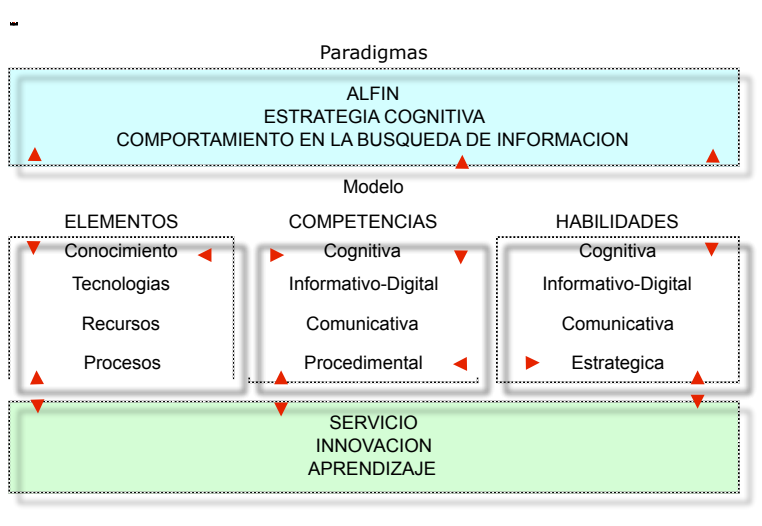

Fig. 1. El modelo ALFINTRA

EI modelo INFOLITRANS parte de la realización de estudios diagnósticos con el colectivo de estudiantes, profesorado experto y profesionales, con la intención de recabar información verdaderamente aplicada en torno a las habilidades y necesidades de ALFIN por parte de esta comunidad de práctica

\section{El portal de contenido ALFINTRA como iniciativa formativa pionera}

Acorde con los procesos de innovación docente y de aplicación de las nuevas metodologías docentes, hemos apostado por el diseño, desarrollo y uso de los portales de contenido educativo, como espacios virtuales que ofrecen múltiples servicios a los miembros de la comunidad universitaria. Constituyen un nodo integrado para el acceso comprensivo y útil de la información y ofrecen, además de contenidos, información, instrumentos para la búsqueda de datos, recursos didácticos, materiales docentes interactivos, herramientas para la comunicación interpersonal, formación, asesoramiento, etc. (Eisler, 2000). De esta forma favorece la creación de comunidades de aprendizaje y espacios académicos, que permitan redefinir en profundidad las relaciones entre profesorado, estudiantado e instituciones (Jafari y Sheehan, 2003).

El modelo Infolitrans en su faceta aplicativa se concreta en el Portal educativo ALFINTRA, dirigido al aprendizaje global de los estudiantes y 
graduados, y centrado en el entrenamiento y adquisición de habilidades, competencias y destrezas genéricas, relacionadas con todos los aspectos importantes de la gestión, acceso y uso de la información (Pinto, Sales, 2009). Para el diseño de contenidos se tomó como base tanto la experiencia docente, como los resultados de investigaciones realizadas en el contexto de la documentación aplicada a la traducción (Pinto \& Sales 2007; Pinto \& Sales 2008a, 2008b; Sales 2008, Pinto, 2009). En ellas se evidencia las diferencias entre el alumnado de primer ciclo, que reconocía que todavía tiene mucho que trabajar en la construcción estratégica de sus hábitos informacionales, lo que provoca considerables desfases entre la necesidad de información, las expectativas y el nivel de logro en la solución del problema, y el alumnado de segundo ciclo, que demostraba ya una progresión resolutiva considerable en cuanto al acceso, uso y aprovechamiento de la información. Observando en conjunto los resultados del cuestionario y las entrevistas focus groups, resulta de interés destacar los aspectos que han orientado el diseño del portal ALFINTRA:

- El alumnado de primer ciclo de la licenciatura de Traducción e Interpretación carece de estrategias claras a la hora de hacer frente a necesidades de información y búsquedas para la resolución de problemas. Acude mayoritariamente a Internet, careciendo de una base sólida de conocimiento sobre el mismo, y realizando búsquedas poco o nada estructuradas, intuitivas, mediante los buscadores más conocidos. Reconoce que necesita de un mayor conocimiento acerca del abanico de fuentes de información para la actividad traductora, así como, en suma, de competencia procedimental y estratégica (capacidad de organización y planificación) y competencia informativo-digital (capacidad de gestión de la información y conocimientos de informática). En definitiva, percibe su propia competencia informacional como endeble y es consciente de la necesidad de trabajar para hacerla crecer, enfatizando de forma especial la necesidad de aprender estrategias crítico-evaluativas en la búsqueda y tratamiento de la información, y la importancia de aprender cómo gestionar la información para construir su propia base de conocimientos.

- El alumnado de segundo ciclo de la licenciatura de Traducción e Interpretación posee cierta habilidad estratégica para hacer frente a necesidades de información y búsquedas para la resolución de problemas. Acude mayoritariamente a Internet, pero ya demuestra cierto nivel de conocimiento sobre el mismo, emple- ando buscadores y sus opciones avanzadas, o acudiendo directamente a fuentes electrónicas precisas que conoce y le aportan garantías de calidad. No obstante, sigue reconociendo que necesita de un mayor conocimiento acerca del abanico de fuentes de información para la actividad traductora, así como, en suma, de competencia procedimental y estratégica (capacidad de organización y planificación) y competencia informativo-digital (capacidad de gestión de la información y conocimientos de informática). Percibe una progresión positiva en cuanto a su propia competencia informacional, pero sigue siendo consciente de la necesidad de trabajar para hacerla crecer.

En definitiva, y si nos remitimos a las tres categorías apuntadas por Maybee (2006) -fuentes de información, procesos de información y base de conocimientos-, cabría decir que, a tenor de los resultados de nuestra investigación previa, el alumnado de primer ciclo necesita refuerzo en todo caso, pero en su percepción apunta esencialmente a la necesidad de conocimiento de fuentes, comienza a ser consciente de la necesidad de entender la búsqueda, recuperación y gestión informativa como parte de un proceso en el que lo esencial es la perspectiva crítica que aporte calidad al resultado final, y todavía no se muestra consciente de la importancia de crear y mantener una propia base de conocimientos. Por su parte, el alumnado de segundo ciclo demuestra tener mayor conocimiento del abanico de fuentes de información y a ser claramente consciente de los elementos del proceso de información, aunque reconozca necesitar refuerzo para su logro. Con respecto a la base de conocimientos, se muestra consciente de su relevancia, pero todavía le falta reforzar la competencia estratégico-procedimental para optimizar todas sus tareas documentales.

Desde el convencimiento de la necesidad de aportar una mayor formación al estudiante de Traducción e Interpretación en la adquisición de una competencia documental ágil y actualizada, se concibe este portal como recurso pionero también para la comunidad profesional y para la formación continua, con el objetivo de lograr estudiantes competentes, entrenados en el hábito de saber explorar los caminos de la información, de reconocer sus cualidades, bondades y debilidades, saber buscar con atino, saber evaluar y seleccionar la información pertinente, saber integrar la información nueva para generar conocimiento y saber usarla debidamente.

Siguiendo la filosofía constructivista pedagógica y las lecciones aprendidas en la ejecución del portal ALFINEEES, ALFINTRA se organiza en 
estas categorías, acompañadas de una ficha tipo que incluye objetivos, competencias a adquirir, actividades a desarrollar, recursos.... (Figura 2).

\section{Fundamentos ALFINTRA}

- Alfabetización informacional en traducción e interpretación (ALFINTRA)

- Acceso y recuperación de información

- Análisis y síntesis

- Evaluación crítica de recursos informativos

- Gestión de la información

- Comunicación y presentación información

- Ética y calidad en el uso de la información

- Recursos especializados por itinerarios

Figura 2.

\section{INFOLITRANS como herramienta para medir la ALFIN}

En el proceso de alfabetización informacional (ALFIN) la evaluación constituye un eje medular y puede abarcar distintos aspectos como la evaluación de sesiones de trabajo en ALFIN, la evaluación de la labor formativa de los bibliotecarios y el profesorado dentro del proceso, la evaluación de programas de ALFIN o la evaluación del aprendizaje de los estudiantes.

Lindauer (2004) señala tres ámbitos de la educación superior en los que se pueden obtener datos para la evaluación, como son el entorno de aprendizaje (el programa, las experiencias extracurriculares y el aprendizaje independiente del estudiante), los componentes del programa de alfabetización informacional y los resultados del aprendizaje, centrado en el rendimiento del estudiante. Estos ámbitos se solapan y se entrecruzan en algunos aspectos y han de entenderse en un contexto formativo amplio.

En la herramienta INFOLITRANS centramos nuestra atención en la evaluación de los resultados del aprendizaje del estudiante en cuanto al conocimiento y dominio de la competencia informacional, teniendo presente que seria recomendable aplicarla tanto al comienzo del proceso formativo como al final, con el fin de correlacionar los objetivos de aprendizaje con los resultados de aprendizaje, midiendo los logros: conocimiento de las competencias adquiridas, las habilidades desarrolladas y los valores y actitudes conseguidos Dugan \& Hernon (2002).
Para el desarrollo de esta herramienta se tuvieron en cuenta la experiencia de algunos trabajos de evaluación de ALFIN, como entre otros el de Dunn (2002) quien diseñó un plan de evaluación plurianual en varias fases para la Universidad del Estado de California; el de Larkin \& Pines (2005) que midieron los resultados de aprendizaje en ALFIN a partir de los cursos introductorios de Psicología en el Colegio Canisius, centrados básicamente en la búsqueda de información en bases de datos, y el trabajo de Markless (2007) que desarrolló un modelo práctico de ALFIN desde la perspectiva del aprendizaje, orientado al personal académico y a los bibliotecarios, en el que enfatiza el proceso de transformación y construcción de conocimiento animando a que los estudiantes sean críticos e independientes, que construyan sus propias perspectivas y visiones en torno al proceso de aprendizaje e investigación. El modelo consta de estas tres etapas, que a su vez agrupan otras subetapas: conexión con la información (entorno, exploración, enfoque, localización...); interacción con la información (pensamiento crítico; análisis y síntesis, transformación, evaluación y construcción) y uso de la información (difusión, comunicación, aplicación).

Asimismo se han revisado algunos cuestionarios empleados para evaluar las habilidades de ALFIN, como el lowa Test of Educational Development (1988), empleado para conocer las destrezas necesarias en el manejo de amplias fuentes de información; el Test of Achievement and Proficiency (1990), empleado para medir la capacidad de los estudiantes para utilizar fuentes de información conocidas; la encuesta "National Survey of Student Engagement" (NSSE) desarrollada por la Indiana University siguiendo las normas $A C R L$, para recoger información sobre la participación de los estudiantes universitarios en los programas y actividades académicas y de aprendizaje; el test SAILS (Standardized Assessment of Information Literacy Skills) aprobado en 2003 por ARL y basado en las normas ALFIN de ACRL, mide en línea las destrezas generales en alfabetización informacional de diversos grupos de estudiantes. Valora el conocimiento de cada encuestado acerca de ALFIN, esto es, lo que las personas saben de forma general acerca de la competencia informacional y no tanto su práctica alfabetizadora, es decir lo que realmente saben hacer. Consta de 45 preguntas seleccionadas al azar de un banco pruebas de más de 250 elementos; el test Education Testing Service (ETS, 2008), conocido hoy por iSkills fue elaborado por la Universidad del Estado de California con el propósito de medir siete aspectos relacionados con la competencia informativo-digital; la en- 
cuesta australiana ISS (Information Skills Survey) (Catts, 2005), que a modo de autoinforme pide al encuestado que describa lo que hace con la información, ofreciendo evidencias de lo que realiza en la práctica. Como fuente de apoyo, la herramienta se sustenta en los resultados derivados de la implementación del cuestionario ALFIN-HUMASS, aplicado al ámbito de Humanidades y Ciencias Sociales (Pinto, M., 2007, 2009), centrado en el autodiagnóstico y evaluación del dominio de la competencia informacional de los estudiantes universitarios en distintas títulos de grado de varias universidades españolas (Psicología, Traducción, Documentación, Ciencias de la Educación...), desde una triple perspectiva: importancia, satisfacción y entorno de aprendizaje en el que el estudiante ha adquirido las competencias.

Es un prototipo web en código abierto, integrado por cuestionarios, tests, resolución de casos específicos para cada una de las competencias estratégicas, informacionales, tecnológicas y sociales que necesita conocer un estudiante universitario de Traducción e Interpretación, como comunidad de aprendizaje específica a la que se dirige el presente proyecto, partiendo de la base de que del abordaje de un colectivo específico y los logros aplicados que puedan derivar de ello, la reflexión teórica y metodológica puede ser más sólida y potencialmente enriquecedora para seguir trabajando en otros contextos. Como objetivos específicos de este prototipo resaltamos los siguientes:

1. Contribuir a la concienciación, por parte de la comunidad estudiantil, de la necesidad de adquirir las competencias genéricas y especialmente todas aquellas relacionadas con el desarrollo de habilidades informacionales y tecnológicas útiles para el aprendizaje.

2. Servir de complemento y de retroalimentación al portal de contenidos ALFINTRA, centrado en el aprendizaje de la práctica alfabetizadora, a través de una oferta formativa especializada, compatible con las propuestas consensuadas por los centros y departamentos de todo el país para los futuros licenciados en traducción e interpretación, teniendo en cuenta la enorme relevancia de la competencia documental en este grado

3. Proporcionar una herramienta electrónica que aporte información específica y personalizada sobre necesidades en competencias estratégicas, informacionales, tecnológicas y sociales por áreas de especialidad, por perfiles y por niveles o itinerarios formativos.

4. Identificar las necesidades de formación en dichas competencias para la producción de materiales que puedan ser utilizados en el aprendizaje virtual y presencial.

La herramienta se estructura en cuatro grandes áreas:

- Búsqueda de información

- Evaluación de la información

- Tratamiento de la información

- Comunicación y difusión de la información

Así, la competencia de búsqueda tiene que estar referida a la recuperación en bases de datos, catálogos automatizados y motores de búsqueda. En segundo lugar, la evaluación de la información tiene que atender a las peculiaridades de la Internet, que ofrece una información riquísima, pero desbordante, y democrática, pero necesitada de contrastación cuidadosa. En tercer lugar, el tratamiento de la información, sin descuidar en absoluto los procesos intelectuales de análisis y síntesis, debe hacer uso intensivo de las tecnologías adecuadas, especialmente de los gestores de bases de datos y los procesadores de información factual, textual y gráfica. Finalmente, la comunicación y difusión de la información debe enseñarse no sólo en sus aspectos psicosociales, sino también tecnológicos, con especial atención a la edición de textos, presentaciones y multimedia, y al aprovechamiento de la Web 2.0.

A grandes rasgos el funcionamiento del prototipo es el siguiente (Infolitrans, 2009):

El alumno identifica una laguna en sus conocimientos, que se puede objetivar como una necesidad de información, e inicia el comportamiento de búsqueda de la información. Para ello, necesita conocer fuentes de información y cómo interrogarlas. Estamos en la fase de búsqueda de información.

Una vez obtenidos los recursos de información relevantes, debe ser capaz de evaluar su calidad y seleccionar los mejores recursos. Se trata de la fase de evaluación de la información.

A la vez, debe codificar ese conocimiento en información mediante herramientas de tratamiento de la información organizando, clasificando y resumiendo los recursos con ayuda de un programa de gestión bibliográfica para su control y utilización futura; extrayendo contenidos y datos; y sistematizándolos mediante bases de datos, hojas de cálculo y programas estadísticos. Como resultado del análisissíntesis de los datos y de su tratamiento y organización en bases de datos y ficheros semiestructurados, el estudiante alcanza un nuevo estado de conocimiento respecto a la fase inicial 
de información. Se trata de la fase de tratamiento de la información.

Por fin, el alumno tiene que comunicar el conocimiento adquirido oralmente y mediante documentos de forma adecuada a la audiencia. En particular, tras un proceso de organización de la información, de síntesis y de análisis de sus objetivos comunicacionales, debe ser capaz de redactar documentos orientados al trabajo en grupo dentro de proyectos y organizaciones, a la divulgación, a la comunicación académica y a la enseñanza. $Y$ debe ser capaz de hacerlo en cuantos más idiomas mejor y con el uso de las tecnologías de la información y la comunicación.

El prototipo INFOLITRANS-Test consta de dos cuestionarios auto-corregibles con informes globales y parciales de cada una de las competencias. El sistema ofrece cuatro cuestionarios con dos niveles - básico y avanzado- y dos modalidades de utilización: evaluación y aprendizaje. El test es cumplimentado online por el estudiante y es evaluado por el sistema con respuestas tipo, ofreciéndole un informe detallado de los resultados obtenidos y de la posible propuesta formativa. Esa interacción del estudiante con el sistema permitirá detectar las necesidades y expectativas sobre cada competencia y proponer un itinerario de autoformación accesible y multinivel, que le ayude en la adquisición, desarrollo y entrenamiento de las competencias informacionales y tecnológicas fundamentales para el aprendizaje a lo largo de la vida.

Para proporcionar una retroalimentación rápida y automatizada se optó por proponer tests de opción múltiple. Y para reducir la carga cognitiva de los estudiantes se optó por un modelo de cuatro ítems y una sola pregunta correcta; de manera que el estudiante se familiarizara rápidamente con el formato del test y se centrara en resolver los contenidos propuestos.

El test es escalable en cuanto al número de preguntas potenciales, de manera que, utilizando un mecanismo aleatorio, el sistema propone tests distintos a diferentes alumnos y a un alumno en diferentes estadios de su formación, moderando artefactos como el reconocimiento de las preguntas anteriores o el comentario previo con estudiantes que han realizado el test previamente. De hecho, el prototipo dispone ya de un mínimo de tres preguntas para cada ítem del test y para cada uno de sus niveles (seis preguntas por ítem, tres por nivel).

El interfaz de usuario es amigable y sencillo. La pantalla de entrada al sistema proporciona una presentación del prototipo, un módulo de registro para los usuarios, el acceso a los cuestiona- rios y los datos de contacto. Las preguntas se presentan situadas en su competencia correspondiente y se puede navegar por las cuatro competencias y por las diferentes preguntas para cada una de ellas, de manera que el estudiante pueda desplazarse entre ellas y contestarlas en el orden que desee y repasarlas posteriormente. Conforme van siendo respondidas las preguntas, van quedado marcada en la pantalla, para que el estudiante lleve un control de las que ha contestado.

Finalmente, el sistema ofrece como resultado un informe detallado para el estudiante tanto de aprendizaje como de evaluación, que ofrece un diagnóstico sobre su situación, sus errores y aciertos y le propone recursos de calidad y alternativas para el aprendizaje.

\section{Conclusiones}

Tanto la elaboración del portal ALFINTRA como de la herramienta INFOLITRANS fomentará la posibilidad de implementar mucho mejor las TICs a nivel docente en la materia que nos ocupa, constituyéndose en plataforma e-learning las ventajas de su aplicación son significativas pues facilita:

- Ayuda al estudiante para medir su nivel de competencia informacional, detectando las áreas en las que precisa mejorar y ofreciéndole recursos para lograr dicho objetivo

- Seguimiento de los resultados de aprendizaje por parte del estudiante

- Personalización en tiempo real de cuestionarios e información

- Comparativa con respecto a otros miembros del mismo perfil de usuario y la posibilidad de realizar estudios estadísticos y/o publicaciones

\section{Referencias}

ACRL, Information literacy competency standards for higher education. 2000. http://www.ala.org/ala/acrl/acrlstan dards/standards.

ANECA. Informe final del Trabajo de diseño del plan de estudios y título de grado en Traducción e Interpretación, 2004. http://www.aneca.es/modal_eval/docs/libroblan co_traduc_borrador.pdf.

Catts, R. Information Skills Survey. Technical Manual. Camberra: CAUL, 2005.

Delors, J. (coord). La educación encierra un tesoro. Madrid: Santillana, 1996

Dunn, K. Assessing information literacy skills in the California state university: a progress report. // Journal of Academic Librarianship. 28:1/2 (2002) 26-35 
Eisler, D: The portal progress: A gateway for access, information and learning communities. // Syllabus. 14:2 (2000)12-18.

ETS. iSkills Assessment. 2008. Disponible en http://www.ets.org/portal/site/ets/menuitem.1488512ecfd 5b8849a77b13bc3921509/?vgnextoid=159f0e3c27a851 10VgnVCM10000022f95190RCRD\&vgnextchannel=e5b 2a79898a85110VgnVCM10000022f95190RCRD

Hernon, P.; Dugan, R.; Schwartz, C. Revisiting Outcomes Assessment in Higher Education. Westport, CT: Libraries Unlimited, 2006

Hurtado, A. La enseñanza de la traducción directa 'general'.Objetivos de aprendizaje y metodología. // A. Hurtado Albir (ed.). La enseñanza de la traducción, Castellón: Universitat Jaume I. Collecció Estudis sobre la Traducció, 1996. 3, 31-56.

Infolitrans Test: Proyecto de Estudio y Análisis financiando por el Ministerio de Educación y Ciencia, 2008-09. Coord. Maria Pinto. www.infolitrans.edu.es.

Jafari, A; Sheehan, M. Designing Portals: Opportunities and Challenges. Hershey: Information Science Publishing, 2003.

Larkin, J. E; Pines, H. A. Developing information literacy and research skills in introductory Psychology: a case study. Journal of Academic Librarianship. 35: 1 (2005) 40-45.

Lindauer, B. G. Assessing Community Colleges: Information Literacy Competencies and Other Library Services and Resources. $2004 . \quad$ http://fog.ccsf.cc.ca.us/ bg ratch/assess.html.

Markless, S; Streatfield, D. R. Three decades of information literacy: redefining the parameters. En: Andretta, S. Change and challenge: information literacy for the 21st century (pp. 15-36). Adelaide: Auslib Press, 2007

Maybee, C.: Undergraduate perceptions of information use: The basis for creating user-centered student Information Literacy Instruction. // The Journal of Academic Librarianship. 32:1 (2006) 79-85.

National Survey of Student Engagement. http://nsse.iub.edu/index.cfm

OCDE (2005). The definition and selection of key competencies. Executive Summary, https://www.pisa.oecd.org/ dataoecd/47/61/35070367.pdf (7 de mayo de 2009).

Pinto, M. Quality Factors in Documentary Translation. Meta. Evaluation: Paramètres, méthodes, aspects pedagogiques. 46:2 (2001) 288- 300.

Pinto, M. Design of the IL-HUMASS Survey on Information Literacy in Higher Education: a self-assessment approach. // Journal of Information Science. (2009, en prensa)

Pinto, M. La alfabetización en información para el aprendizaje virtual en el área de Humanidades en el marco del EEES. Proyecto de investigación financiado por Secretaria de Estado de Universidades e Investigación. Ministerio de Educación y Ciencia, 2007-2008.

Pinto, M.; García Marco, F. J. La enseñanza-aprendizaje de las competencias genéricas en el Espacio Europeo de Educación Superior: el proyecto ALFINEEES. // Innovación docente, tecnologías de la información y la comunicación e investigación educativa en la Universidad de Zaragoza: caminando hacia Europa. Zaragoza: Universidad, 2007. p. $62-63+32$ p. en CD. http://www.unizar.es/eees/innovacion06/capitulo2.html

Pinto, M.; Sales, D: El aprendizaje basado en recursos electrónicos: ALFINTRA un portal de contenido para la documentación aplicada a la traducción. // Documentación aplicada y Espacio Europeo de Educación Superior. Madrid: Arco/Libro, 2009

Pinto, M.; Sales, D. Towards User-centred Information Literacy Instruction in Translation: The View of Trainers. // The Interpreter and Translator Trainer. 2:1 (2008) 47-74.
Pinto, M.; Sales, D. INFOLITRANS: A Model for the Development of Information Competence for Translators. /I Journal of Documentation. 64:3 (2008) 413-437.

Project SAILS: Standardized Assessment of Information Literacy Skills. http://www. projectsails.org.

Sales, D. Documentación aplicada a la traducción: Presente y futuro de una disciplina. Trea, Gijón, 2006

Vienne, J. Which competences should we teach to future translators, and how?. // C. Schäffner y B. Adab (eds.): Developing Translation Competence, John Benjamins. Ámsterdam/Filadelfia: 91-100, 2000. 